\title{
Changed concepts and definitions of myeloproliferative neoplasms (MPN), myelodysplastic syndromes (MDS) and myelodysplastic/myeloproliferative neoplasms (MDS/MPN) in the updated 2008 WHO classification
}

\author{
Konnie M. Hebeda • F. Fend
}

Received: 28 August 2009 / Accepted: 3 September 2009 /Published online: 26 September 2009

(C) Springer-Verlag 2009

\begin{abstract}
The purpose of this overview is to discuss the changes in the 2008 WHO classification of myeloid neoplasms, with exclusion of acute myeloid leukaemia. Specific mutations or rearrangements leading to constitutive activation of growth factor receptors or cytoplasmic tyrosine kinases are now recognised as recurrent genetic events characterising the group of myeloproliferative neoplasms (MPN). A newly introduced subgroup consists of patients with persistent eosinophilia and myeloid or lymphoid proliferations harbouring specific genetic changes involving platelet-derived growth factor receptors alpha and beta (PDGFRA and PDGFRB) or fibroblast growth factor receptor 1 (FGFR1). The clinical relevance of recognising myeloid neoplasms with aberrant tyrosine kinase activity is based in novel treatment options with tyrosine kinase inhibitors. The myelodysplastic syndromes (MDS) without increased blasts are further divided into subtypes of refractory cytopaenias with unilineage dysplasia. A new provisional entity is refractory cytopaenia of childhood. Down syndrome- and therapy-related myeloid neoplasms, including MDS, were moved to the section of acute myeloid leukaemia and related precursor neoplasms.
\end{abstract}

Keywords Review Classification · Myelodysplastic syndrome $\cdot$ Myeloproliferative neoplasm $\cdot$ Updated WHO classification

\footnotetext{
K. M. Hebeda $(\bowtie)$

Department of Pathology, University Nijmegen Medical Center, P.O. Box 9101, 6500 HB Nijmegen, The Netherlands e-mail:k.hebeda@pathol.umcn.nl

F. Fend

Institute of Pathology, University Hospital Tübingen,

Tübingen, Germany
}

\section{Introduction}

Since the publication of the third edition of the WHO classification in 2001 several fundamental changes in the understanding of myeloid neoplasms have occurred. The conceptual change in classifying myeloid disorders, from a morphology-based to an integrative approach with strong emphasis on recurrent cytogenetic and molecular aberrations, which was initiated for the acute leukaemias in the 2001 WHO classification, now extends to many of the chronic myeloproliferative disorders. The discovery of activating point mutations in tyrosine kinases, starting with JAK2 V617F in Ph- myeloproliferative neoplasms, especially polycythemia vera, led to a unifying concept of growth receptor tyrosine kinase activation involved in the aetiology of myeloproliferative neoplasms (MPN). This explains the inclusion of mastocytosis in the group of MPN in the updated WHO classification [1].

A new category in the 2008 classification is the group of patients with unexplained persistent eosinophilia, previously grouped as hypereosinophilic syndrome or chronic eosinophilic leukaemia. In most of these patients, specific genetic changes involving platelet-derived growth factor receptor alpha (PDGFRA), platelet-derived growth factor receptor beta (PDGFRB) or fibroblast growth factor receptor (FGFR1) can be demonstrated. This group is now defined by the genetic aberration, in combination with its clinical presentation with myeloid or rarely, lymphoid proliferations. This categorization reduces the spectrum of myelodysplastic/myeloproliferative neoplasm (MDS/MPN) overlap diseases, since a subset of cases of chronic myelomonocytic leukaemia (CMML), but also some cases of atypical chronic myelogenous leukaemia (aCML) and juvenile myelomonocytic leukaemia (JMML) now can be assigned to this new group. The rationale for 
separating these cases with aberrant tyrosine kinase activity from other myeloid neoplasms lies again in the new treatment options with tyrosine kinase inhibitors.

In the previous WHO classification of myelodysplastic syndromes (MDS) a significant number of patients could not be classified because they did not fulfil the minimum requirements of at least bilineage dysplasia and cytopaenia, if the erythroid lineage was not affected. For these cases, subtypes of refractory cytopaenias with unilineage dysplasia were now introduced. Furthermore, the diagnosis of refractory cytopaenia with multilineage dysplasia (RCMD) required at least bicytopaenia and a two-lineage dysplasia. In the new classification, a single cytopaenia in combination with a bilineage dysplasia is sufficient for this diagnosis. The distinction of RCMD with or without ring sideroblasts is no longer incorporated in the 2008 classification. A newly introduced provisional entity is refractory cytopaenia of childhood for all juvenile MDS cases without increased blasts.

The additional diagnostic and prognostic value of bone marrow biopsies, especially for BCR-ABL negative MPN and MDS is acknowledged in the updated WHO classification and a semi-quantitative grading of fibrosis is proposed. Flow-cytometric immunophenotyping is not yet recognised as an obligatory diagnostic tool for any of the myeloid neoplasms.

In this review, the changes in definition of the myeloid neoplasms with myeloproliverative and/or dysplastic features, with the exception of acute myeloid leukaemia will be presented.

\section{Myeloproliferative neoplasms}

The group of the chronic myeloproliferative diseases, now called myeloproliferative neoplasms (MPN), is extended by the inclusion of mastocytosis (see Table 1). The diagnostic criteria are unchanged for chronic myelogenous leukaemia (CML), BCR-ABL1-positive, chronic neutrophilic leukaemia (CNL) and myeloproliferative neoplasm, unclassifiable (MPN, U). All other disease entities underwent major or minor revisions, mainly due to new genetic insights enabling more strict disease definition. The most significant impact on the classification of MPN was the discovery of the activating point mutation $\mathrm{V} 617 \mathrm{~F}$ in the pseudokinase domain of the janus kinase 2 (JAK2) which leads to independence from exogenous hematopoietic growth factors due to constitutive signalling in the JAK-STAT pathway, and results in overproduction of mature blood cells, so characteristic for MPN. However, it has to be taken into account that the absence of a JAK2 mutation does not exclude a diagnosis of either PMF or ET, and that in the presence of the mutation, a proper disease categorization still requires a haematological work-up including a trephine BM biopsy. Nevertheless, inclusion of the JAK2 V617F
Table 1 Myeloproliferative neoplasms, new names and changed criteria

\begin{tabular}{|c|c|}
\hline WHO 2008 & WHO 2001 \\
\hline $\begin{array}{l}\text { Chronic myelogenous leukaemia, } \\
\text { BCR-ABL-1 positive }\end{array}$ & $\begin{array}{l}\text { Chronic myelogenous } \\
\text { leukaemia }\end{array}$ \\
\hline Chronic neutrophilic leukaemia & Unchanged \\
\hline $\begin{array}{l}\text { Polycythaemia vera } \\
-J A K 2 \text { V617F or exon } 12 \text { mutation }\end{array}$ & Unchanged \\
\hline $\begin{array}{l}\text { Primary myelofibrosis } \\
\text { - JAK2 (50\%) or MPL (5\%) mutation }\end{array}$ & $\begin{array}{l}\text { Chronic idiopathic } \\
\text { myelofibrosis }\end{array}$ \\
\hline $\begin{array}{l}\text { Essential thrombocythaemia } \\
\text { - Thrombocytes }>450 \times 10^{9} / \mathrm{L}\end{array}$ & $\begin{array}{l}\text { Unchanged } \\
\text { - Thrombocytes } \\
>600 \times 10^{9} / \mathrm{L}\end{array}$ \\
\hline \multicolumn{2}{|l|}{ - JAK2 mutation (50-60\%) } \\
\hline Chronic eosinophilic leukaemia, NOS & $\begin{array}{l}\text { Chronic eosinophilic } \\
\text { leukaemia }\end{array}$ \\
\hline \multicolumn{2}{|l|}{$\begin{array}{l}\text { - No BCR-ABL1, PDGFRA, PDGFRB } \\
\text { or FGFR1 translocation }\end{array}$} \\
\hline Mastocytosis & $\begin{array}{l}\text { not included in this } \\
\text { category before }\end{array}$ \\
\hline \multicolumn{2}{|l|}{ - KIT mutation $(>90 \%)$} \\
\hline $\begin{array}{l}\text { Myeloproliferative neoplasm, } \\
\text { unclassifiable }\end{array}$ & $\begin{array}{l}\text { Chronic myeloproliferative } \\
\text { disease, unclassifiable }\end{array}$ \\
\hline
\end{tabular}

mutation (or JAK2 exon 12 mutations in PV) into the diagnostic criteria of MPN has simplified diagnosis for cases with borderline hematologic changes which previously required extensive work to exclude a reactive increase in peripheral blood cell counts.

\section{Chronic eosinophilic leukaemia}

In the new WHO classification chronic eosinophilic leukaemia (CEL), not otherwise specified, is a limited group of cases with a clonal proliferation of eosinophil precursors resulting in persistent eosinophilia as dominant hematologic abnormality, in which no specific genetic gene rearrangement including $B C R-A B L 1, P D G F R A, P D G F R B$ or FGFR1 is present. Cases with a PCM1-JAK2 rearrangement, previously called atypical CML, are suggested to be included in this category. The clinical and morphological criteria were not changed. For cases without demonstration of clonality, idiopathic hypereosinophilic syndrome remains the main differential diagnosis.

\section{Polycythaemia vera}

Polycythaemia vera (PV) now only requires the presence of the JAK2 V617F mutation or an equivalent mutation in exon 12 , in addition to increased haemoglobin $(>18.5 \mathrm{~g} / \mathrm{dL}$ in men, $16.5 \mathrm{~g} / \mathrm{dL}$ in women) and either panmyelosis in a 
bone marrow biopsy, a low serum erythropoietin level, or endogenous erythroid colony formation [2]. This allows an earlier diagnosis, often before the onset of obvious splenomegaly or leukocytosis, and obviates red blood cell mass measurement. In addition, the description of a prepolycythaemic phase with thrombocytosis that occurs in up to $15 \%$ of the patients and clinically mimics ET is added. A bone marrow trephine should be performed mainly for grading of fibrosis.

\section{Primary myelofibrosis}

In the revised classification, the diagnostic criteria of primary myelofibrosis (PMF) are independent of disease stage and the presence of fibrosis at diagnosis. They include now detection of a $J A K 2$ V617F (approx. $50 \%$ ) or $M P L$ (5\%) mutation, which obviates extensive exclusion of reactive causes of fibrosis. A semi-quantitative grading of bone marrow fibrosis ranging from MF-0 (normal) to MF-3 (osteosclerosis) is adopted [3].

\section{Essential thrombocythaemia}

Although the diagnosis of essential thrombocythaemia (ET) still requires exclusion of all other causes of sustained thrombocytosis, the diagnostic platelet count level is lowered from 600 to $450 \times 10^{9} / \mathrm{L}$ and a $J A K 2$ or $M P L$ mutation, if present (JAK2 in about $50 \%$ ), now can serve as a positive criterion. For the rare event of post-ET myelofibrosis, diagnostic criteria are given, intending to help distinguish post-ET myelofibrosis from PMF. Whether also pre-fibrotic PMF, which shows a worse prognosis in studies based on WHO criteria, and ET can be safely distinguished on morphological grounds remains a matter of debate.

\section{Mastocytosis}

Mastocytosis was previously considered a haematopoietic disorder that was not grouped under the myeloproliferative disorders, since its clonal, neoplastic nature could usually not been proven. Due to the recognition of activating point mutations in the KIT gene, typically $\mathrm{D} 816 \mathrm{~V}$, it is now grouped under the MPN. The diagnostic criteria were not changed, and all provisional entities, consisting of bone marrow mastocytosis and smouldering systemic mastocytosis as subtypes of indolent systemic mastocytosis (SM), lymphadenopathic mastocytosis with eosinophilia (if PDGFRA rearrangement is excluded) as part of aggressive mastocytosis, and SM with associated clonal haematological non-mast-cell lineage disease (SM-AHNMD), are now accepted as definitive variants. Of clinical importance is the resistance of the KIT D816V mutation to the kinase inhibitor imatinib.
Myeloid and lymphoid neoplasms with eosinophilia and abnormalities of PDGFRA, PDGFRB or FGFR1

A completely new group of diseases, defined by their shared genetic defect, leading to an aberrant expression of PDGFRA, $P D G F R B$ or FGFR1, was compiled from former categories of myeloid neoplasms and lymphoid neoplasms with features of MPN (Table 2). These are all rare diseases, usually presenting with eosinophilia and variable clinical features that are influenced by the partner gene of the rearranged growth factor receptor. Since the cell of origin is a mutated multi- or pluripotent stem cell, the involved cell lineage can range from myeloid to lymphoid or mast cells and combinations of these. These neoplasms are sensitive to tyrosine kinase inhibitors. Acute transformations are usually of the myeloid lineage.

\section{Myeloid and lymphoid neoplasms with PDGFRA rearrangement}

Neoplasms with PDGFRA rearrangement present in most cases as a chronic eosinophilic leukaemia with a cryptic deletion at $4 \mathrm{q} 12$ (including the $C H I C 2$ gene) that leads to a FIP1L1-PDGFRA fusion gene. Splenomegaly, increased serum tryptase and a marked elevation of serum vitamin B12 are common, and trephine biopsies can show an increase of sometimes atypical mast cells. Because of these features, such cases previously had commonly been diagnosed as systemic mastocytosis with eosinophilia; a term now obsolete due to the molecular alterations separating these two entities. In rare cases, primary presentation as or progression to acute myeloid leukaemia or T-lymphoblastic lymphoma is seen.

\section{Myeloid neoplasms with PDGFRB rearrangement}

Chronic myelomonocytic leukaemia with eosinophilia and $\mathrm{t}(5 ; 12)$ involving the ETV6 gene is the most common presentation of myeloid neoplasms with $P D G F R B$ rearrangement. Variant partner genes can induce clinical pictures of chronic eosinophilic leukaemia, atypical CML or JMML, usually with eosinophilia.

\section{Myeloid and lymphoid neoplasms with FGFR1 abnormalities}

The myeloid and lymphoid neoplasms with FGFR1 abnormalities also known as " $8 \mathrm{p} 11$ stem cell syndrome"

Table 2 Myeloid and lymphoid neoplasms with eosinophilia and abnormalities of PDGFRA, PDGFRB or FGFR1

Myeloid and lymphoid neoplasms with $P D G F R A$ rearrangement

Myeloid neoplasms with $P D G F R B$ rearrangement

Myeloid and lymphoid neoplasms with FGFR1 abnormalities 
due to the chromosomal location of the gene are characterised by a somewhat younger age of onset (around 32 years) and a more variable clinical picture than the other neoplasms in this category. Presentation may be as CEL, AML, lymphoblastic lymphoma or myeloid sarcoma with extramedullary and nodal involvement. Systemic symptoms and peripheral or tissue eosinophilia are common. The WHO classification recommends adding a specification, including information of the major involved cell line in the diagnosis, for example leukaemia/lymphoma associated with FGFR1 rearrangement/myeloid sarcoma.

\section{Myelodysplastic/myeloproliferative neoplasm}

The unifying biological defect in the group of myelodysplastic/ myeloproliferative neoplasms (Table 3) seems to be an aberrancy in the RAS/MAPK signalling pathway, leading to neutrophilic proliferations due to GM-CSF hypersensitivity. However, data are still insufficient for diagnostic or prognostic purposes and the definition of this group remains largely unchanged, except for cases with specific recurrent genetic defects mentioned above that are moved to other categories. The definitions of JMML and myelodysplastic/ myeloproliferative neoplasm, unclassifiable (MDS/MPN, U) have not been changed.

\section{Chronic myelomonocytic leukaemia}

The definition of CMML is only slightly changed by excluding cases with PDGFRA or PDGFRB rearrangement, also resulting in omission of the subgroup CMML with eosinophilia. JAK2 V617F occurs in a minority $(<5 \%)$ of otherwise typical cases. The categorization of CMML into two prognostic groups is based on blast counts, with $<5 \%$ blasts and promonocytes in the $\mathrm{PB}$ and $<10 \%$ in the bone marrow for CMML-1, and 5-19\% blasts and promonocytes in the $\mathrm{PB}$ and $10-19 \%$ in the bone marrow for CMML-2.

\section{Atypical chronic myelogenous leukaemia, BCR-ABL-1 negative}

The addition of "BCR-ABL-1 negative" in the updated WHO classification reflects the somewhat stricter definition of atypical chronic myelogenous leukaemia, $B C R-A B L-1$ negative (aCML), as a proliferation of the neutrophil lineage with exclusion of $B C R-A B L-1, P D G F R$ and $F G F R$ translocations and prominent signs of dysplasia, especially dysgranulopoiesis. Although the JAK2 V617F mutation is still allowed according to the WHO classification, recent results demonstrate that aCML as based on the morphological criteria of the WHO is a JAK2 V617F negative neoplasm [4]. Cases with PCM1-JAK2 translocation are considered as probable CEL.

\section{Refractory anaemia with ring sideroblasts associated with marked thrombocytosis}

Although more cases of refractory anaemia with ring sideroblasts associated with marked thrombocytosis (RARS-T) have been studied since it was accepted as a provisional entity in 2001, its status is not yet determined. Whether these cases are really a unique entity or represent secondary changes in either MDS or MPN is not yet clear. Detection of the JAK2 V617F mutation in about $60 \%$ of cases puts them closer to the MPN category. In a recent compilation of clinical, morphological and molecular data from the literature, the authors convincingly argue that RARS-T might indeed be a variant of ET [5].

\section{Myelodysplastic syndromes}

Myelodysplastic syndromes are characterised by varying combinations of cytopaenias, dysplastic morphology of the myeloid cell lines and cytogenetic aberrancies. Since the third edition that extended a mainly morphological classification by incorporating cytogenetic information for diagnosis (for example MDS with isolated $\operatorname{del}(5 q)$ ) and risk stratification, the biological spectrum of MDS is now more fully acknowledged by adding a new category of refractory cytopaenias with unilineage dysplasia and bi- or unilineage cytopaenia (Table 4).

Even in the absence of dysplasia above the 10\% threshold, persistent cytopaenia can now be diagnosed as MDS, $U$ in the presence of specific cytogenetic abnormalities that include frequent chromosomal losses and translocations. For persistent cytopaenia without cytogenetic changes or dysplasia, the use of 'idiopathic cytopaenia of undetermined significance (ICUS)' is recommended. In any case, exclusion of reactive conditions, for example drug or toxin exposure, infections, immunological disorders or vitamin deficiencies remains essential.

Myeloid proliferations related to Down syndrome and therapy-related myeloid neoplasms, including MDS, are now included in the group of acute myeloid leukaemia and
Table 3 Myelodysplastic/ Myeloproliferative neoplasms
Chronic myelomonocytic leukaemia

Atypical chronic myelogenous leukaemia, BCR-ABL-1 negative

Juvenile myelomonocytic leukaemia

Refractory anaemia with ring sideroblasts associated with marked thrombocytosis (provisional) 
Table 4 Myelodysplastic syndromes

Refractory cytopaenia with unilineage dysplasia

Refractory anaemia with ring sideroblasts

Refractory cytopaenia with multilineage dysplasia

Refractory anaemia with excess blasts

Myelodysplastic syndrome with isolated del $(5 \mathrm{q})$

Myelodysplastic syndrome, unclassifiable

Refractory cytopaenia of childhood

related precursor neoplasms that is not within the scope of this review.

\section{Refractory cytopaenia with unilineage dysplasia}

The new category of refractory cytopaenia with unilineage dysplasia (RCUD) includes uni- or bicytopaenias with dysplasia in more than $10 \%$ of cells of only one lineage in the absence of increased blasts $(<1 \%$ in blood, $<5 \%$ in bone marrow). The dysplastic lineage defines the name. Thus, in refractory anaemia the criteria are slightly altered to allow other cytopaenias in addition to anaemia, as long as there is no dysplasia in more than $10 \%$ of these other cell lines.

Refractory neutropenia and refractory thrombocytopaenia are new additions with dysplasia in at least $10 \%$ of neutrophils or megakaryocytes, respectively. Cases with pancytopaenia and unilineage dysplasia have to be classified as MDS, U. An overview of the classification of MDS without an increase in marrow blasts is given in Table 5 .

\section{Refractory anaemia with ring sideroblasts}

The criteria of refractory anaemia with ring sideroblasts have not been changed, except for allowing bicytopaenia.

\section{Refractory cytopaenia with multilineage dysplasia}

Two changes were introduced for refractory cytopaenia with multilineage dysplasia. First, while at least two cell lines have to be dysplastic, the number of cytopaenias is now irrelevant provided there is at least one cytopaenia. Second, the occurrence of $>15 \%$ ring sideroblasts does no longer require separate mentioning as RCMD-RS since prognosis and other disease characteristics are not different.

\section{Refractory anaemia with excess blasts}

The definition of both categories of refractory anaemia with excess blasts, RAEB-1 and RAEB-2 was not changed, but Auer rods are now considered a definite criterion for RAEB-2, irrespective of the blast percentage.

Hypoplastic MDS and MDS with myelofibrosis are separately discussed since they pose specific problems to diagnose correctly. They are not recognised as defined entities because of lack of significant prognostic impact. Diagnosis usually requires bone marrow biopsy for estimation of blasts, CD34 stain can be especially helpful in this. A temporary definition for RAEB with fibrosis (RAEB-F), in case of more than $5 \%$ blasts in the BM combined with diffuse coarse reticulin fibrosis, is proposed to be able to assess the prognostic impact of fibrosis in future. Usually, an increase of highly dysplastic and often small megakaryocytes is present, making histological distinction from acute panmyelosis with myelofibrosis, with its abrupt clinical onset, and acute megakaryoblastic leukaemia difficult.

\section{Myelodysplastic syndrome with isolated del( $5 q)$}

The myelodysplastic syndrome with isolated $\operatorname{del}(5 q)$ is somewhat more strictly defined by including an obligatory blast count $<1 \%$ in the peripheral blood and absence of Auer rods. Loss of chromosome Y and JAK2 V617F mutation, but no other genetic aberrancies are allowed until more data are available. Since also some patients with additional chromosomal aberrancies, dysplasia of the myeloid lineage or increased blasts seem to benefit from lenalidomide treatment [6], some changes in the genetic definition of this category can be expected in the future.

Table 5 Summary of cytopaenias and dysplasia characteristics in MDS without an increase of marrow blasts

\begin{tabular}{llll}
\hline Dysplasia & Cytopenia(s) & ring sideroblasts & Categories \\
\hline Unilineage & Uni-or bicytopenia & $<15 \%$ & $\begin{array}{l}\text { Refractory cytopenia with unilineage dysplasia (RCUD) including: } \\
\text { - Refractory anaemia (RA) } \\
\end{array}$ \\
& & & - Refractory neutropenia (RN) \\
& & & - Refractory thrombocytopenia (RT) \\
& Pancytopenia & Myelodysplastic syndrome, unclassified (MDS-U) \\
& Uni-or bi- or pancytopenia & $>15 \%$ & Refractory anaemia with ring sideroblasts (RARS) \\
Multilineage (at least 2) & Uni-or bi- or pancytopenia & $<15 \% />15 \%$ & Refractory cytopenia with multilineage dysplasia (RCMD) \\
\hline
\end{tabular}


Myelodysplastic syndrome, unclassifiable

In the prior WHO classification, myelodysplastic syndrome, unclassifiable (MDS, U) has been an ill-defined wastebasket of cases without increased blasts that did not fulfil the required criteria. In the new definition, this diagnosis can be made in patients with all characteristics of RCUD or RCMD, but a slightly higher percentage of blasts in the peripheral blood $(1 \%)$. Cases with pancytopaenia and unilineage dysplasia have also to be classified as MDS, U.

Finally, persistent cytopaenia in the presence of specific cytogenetic abnormalities but in the absence of dysplasia above the $10 \%$ threshold is diagnosed as MDS, U. A more specific classification is recommended whenever this becomes possible during the development of the disease.

\section{Childhood myelodysplastic syndrome}

An entirely new addition to the classification is the definition of childhood MDS (without specifying the age limit). In cases with increased blasts $(2-19 \%$ in PB or $5-$ $19 \%$ in BM) the same criteria for RAEB- 1 and RAEB-2 are suggested as for adults. Because of some specific disease characteristics of MDS without increased blasts in children, and due to uncertainty about the significance of the number of cytopaenias or dysplastic cell lines, one provisional entity 'refractory cytopaenia of childhood (RCC)' is proposed to cover all these remaining cases. In contrast to adult MDS, a hypocellular BM and bi- or pancytopaenia are common in children. Often erythropoiesis is prominent with large islands of immature forms and impaired maturation. Bone marrow biopsy is also very helpful in identifying dysplastic changes in the often sparse megakaryopoiesis. As in adults, dysplasia is required to be present in at least $10 \%$ of cells in one cell line. The clinical and morphological distinction between RCC and inherited BM failure syndromes and aplastic anaemia remains difficult and controversial for the hypocellular cases.

All myeloid proliferations related to Down syndrome, including MDS, are considered a unique entity and now grouped with acute myeloid leukaemia and related precursor neoplasms.

\section{Conclusions}

The updated, fourth edition of the WHO classification reflects the increased knowledge about genetic alterations in myeloproliferative neoplasms and gives a more coherent and systematic classification of myelodysplastic syndromes. Most importantly, we see that this increased understanding of the biologic basis of these disorders translates into therapeutic advances benefitting the patients. Nevertheless, classification of myeloid disorders still requires a multi-faceted rather than a unidirectional approach, including a meticulous clinical and morphological work-up in addition to genetic analysis.

Conflict of interest The authors declare that they have no conflict of interest.

\section{References}

1. Swerdlow SH, Campo E, Harris NL et al (2008) WHO classification of tumours of haematopoietic and lymphoid tissues. IARC, Lyon

2. Tefferi A, Thiele J, Orazi A, Kvasnicka HM, Barbui T, Hanson CA, Barosi G, Verstovsek S, Birgegard G, Mesa R, Reilly JT, Gisslinger H, Vannucchi AM, Cervantes F, Finazzi G, Hoffman R, Gilliland DG, Bloomfield CD, Vardiman JW (2007) Proposals and rationale for revision of the World Health Organization diagnostic criteria for polycythemia vera, essential thrombocythemia, and primary myelofibrosis: recommendations from an ad hoc international expert panel. Blood 110(4):1092-1097 Epub 2007 May 8

3. Thiele J, Kvasnicka HM, Facchetti F, Franco V, van der Walt J, Orazi A (2005) European consensus on grading bone marrow fibrosis and assessment of cellularity. Haematologica 90:1128-1132

4. Fend F, Horn T, Koch I, Vela T, Orazi A (2008) Atypical chronic myeloid leukemia as defined in the WHO classification is a JAK2 V617F negative neoplasm. Leuk Res 32(12):1931-1935 Epub 2008 Jun 13

5. Wardrop D, Steensma DP (2009) Is refractory anaemia with ring sideroblasts and thrombocytosis (RARS-T) a necessary or useful diagnostic category? Br J Haematol 144(6):809-817 Epub 2008 Dec 11

6. Ades L, Boehrer S, Prebet T, Beyne-Rauzy O, Legros L, Ravoet C, Dreyfus F, Stamatoullas A, Chaury MP, Delaunay J, Laurent G, Vey N, Burcheri S, Mbida RM, Hoarau N, Gardin C, Fenaux P (2009) Efficacy and safety of lenalidomide in intermediate-2-or high risk myelodysplastic syndromes (MDS) with $5 \mathrm{q}$ deletion: Results of a phase II study. Blood 113(17):3947-3952 Epub 2008 Nov 5 